\title{
Withaferin A induces apoptosis through the generation of thiol oxidation in human head and neck cancer cells
}

\author{
JONG WON PARK ${ }^{1}$, KYOUNG-JIN MIN ${ }^{2}$, DONG EUN KIM ${ }^{1}$ and TAEG KYU KWON ${ }^{2}$ \\ Departments of ${ }^{1}$ Otolaryngology and ${ }^{2}$ Immunology, School of Medicine, Keimyung University, \\ Dalseo-Gu, Daegu 704-701, Republic of Korea
}

Received June 12, 2014; Accepted October 22, 2014

DOI: $10.3892 /$ ijmm.2014.1983

\begin{abstract}
Withaferin A is a steroidal lactone purified from the Indian medicinal plant, Withania somnifera. Withaferin A has been shown to inhibit the proliferation, metastasis, invasion and angiogenesis of cancer cells. In the present study, we investigated whether withaferin A induces apoptosis in the human head and neck cancer cells, AMC-HN4. Withaferin A markedly increased the sub-G1 cell population and the cleavage of poly(ADP-ribose) polymerase (PARP), which are markers of apoptosis. Pan-caspase inhibitor, z-VAD-fmk (z-VAD), markedly inhibited the withaferin A-induced apoptosis. However, the withaferin A-induced increase in the expression of COX-2 was not affected by treatment with $\mathrm{z}$-VAD. Furthermore, withaferin A upregulated cyclooxygenase-2 (COX-2) expression. The COX-2 inhibitor, NS-398, reduced the withaferin A-induced production of prostaglandin $\mathrm{E}_{2}$. However, treatment with NS-398 did not affect the sub-G1 population and the cleavage of PARP. In addition, the withaferin A-induced apoptosis was independent of reactive oxygen species production. Thiol donors [N-acetylcysteine (NAC) and dithiothreitol (DTT)] reversed withaferin A-induced apoptosis. Therefore, our data suggest that withaferin A induces apoptosis through the mechanism of thiol oxidation in head and neck carcinoma cells.
\end{abstract}

\section{Introduction}

Withaferin A is a steroidal lactone purified from the Indian medicinal plant, Withania somnifera and has been shown to exert anticancer effects in different types of cancer cells, such as prostate cancer (1), myeloid leukemia (2), breast cancer (3) and

Correspondence to: Dr Dong Eun Kim, Department of Otolaryngology, School of Medicine, Keimyung University, 2800 Dalgubeoldaero, Dalseo-Gu, Daegu 704-701, Republic of Korea

E-mail: entkde@dsmc.or.kr

Professor Taeg Kyu Kwon, Department of Immunology, School of Medicine, Keimyung University, 2800 Dalgubeoldaero, Dalseo-Gu, Daegu 704-701, Republic of Korea

E-mail: kwontk@dsmc.or.kr

Key words: withaferin A, cyclooxygenase-2, reactive oxygen species, apoptosis renal cancer (4) cells. The mechanisms responsible for the withaferin A-mediated anticancer effects are multiple. For example, withaferin A has been shown to induce apoptosis through the upregulation of intracellular reactive oxygen species (ROS) generation $(5,6)$, prostate apoptosis response-4 (Par-4) induction (1), p38 MAP kinase activation (7), Akt inactivation (8) and the upregulation of endoplasmic reticulum (ER) stress (4). It has been reported that withaferin A induces apoptosis in head and neck carcinoma cells and inhibits Akt activation (9). However, the cellular and molecular mechanisms underlying withaferin A-induced apoptosis in head and neck carcinoma cells are not yet fully understood.

Cyclooxygenase (COX) converts arachidonic acid into prostaglandin $(\mathrm{PG}) \mathrm{G}_{2}$, which is then reduced to $\mathrm{PGH}_{2}$, a is precursor of other prostanoids. $\mathrm{COX}$ is divided into 2 isoforms. COX-1 is constitutively expressed in the majority of tissues and has physiological functions, whereas COX-2 is induced by inflammatory cytokines (10), the mutation of oncogenes (11) and tumor promoters (12). COX-2 is overexpressed in multiple types of cancer, such as pancreatic (13), colon (14), cervical (15), renal (16) and head and neck (17) cancer. The overexpression of COX-2 has been shown to be associated with the promotion of angiogenesis, invasion and proliferation, and the inhibition of apoptosis. Therefore, the downregulation of COX-2 expression and activity enhances apoptosis $(18,19)$. Furthermore, Limami et al $(20)$ reported that the attenuation of COX-2 expression and COX-2 downregulation by siRNA enhanced apoptosis in ursolic acidtreated colorectal cancer cells. Therefore, the upregulation of COX-2 expression by anticancer drugs may promote resistance to apoptosis; thus, the downregulation of COX-2 expression and activity may enhance susceptibility to apoptosis.

In the present study, we investigated whether the withaferin A-induced COX-2 upregulation is involved in resistance to apoptosis in the human head and neck carcinoma cells, AMC-HN4.

\section{Materials and methods}

Cells and materials. The human head and neck cancer cells, AMC-HN4, were obtained from the Asan Medical Center (Seoul, Korea). The cells were cultured in Dulbecco's modified Eagle's medium that contained $10 \%$ fetal bovine serum, $20 \mathrm{mM}$ HEPES buffer and $100 \mathrm{mg} / \mathrm{ml}$ gentamicin. Withaferin A was purchased from Biomol Research Laboratories, Inc. (Plymouth 
Meeting, PA, USA). z-VAD-fmk (a pan-caspase inhibitor) and NS-398 (a COX-2 specific inhibitor) were purchased from Calbiochem (San Diego, CA, USA). Anti-poly(ADP-ribose) polymerase (PARP) antibodies (sc-25780) were purchased from Santa Cruz Biotechnology, Inc. (Santa Cruz, CA, USA). Anti-actin antibody (A5441) was obtained from Sigma (St. Louis, MO, USA). Anti-COX-2 (Cat\#160106) antibody was purchased from Cayman Chemical Co. (Ann Arbor, MI, USA). N-acetylcysteine (NAC), dithiothreitol (DTT) and all the other chemicals were obtained from Sigma.

Flow cytometric analysis and cell morphology. Approximately $1 \times 10^{6}$ cells were suspended in $100 \mu \mathrm{l}$ PBS, and $200 \mu 195 \%$ ethanol were added while vortexing. The cells were incubated at $4^{\circ} \mathrm{C}$ for $1 \mathrm{~h}$, washed with PBS and resuspended in $250 \mu \mathrm{l} 1,12 \%$ sodium citrate buffer ( $\mathrm{pH} 8.4$ ) together with 12,5 $\mu \mathrm{g}$ RNase. Incubation was carried out at $37^{\circ} \mathrm{C}$ for $30 \mathrm{~min}$. Cellular DNA was then stained by applying $250 \mu \mathrm{l}$ propidium iodide $(50 \mu \mathrm{g} / \mathrm{ml})$ for $30 \mathrm{~min}$ at room temperature. The stained cells were analyzed by fluorescence-activated cell sorting (FACS) on a FACScan flow cytometer (E5464; Becton-Dickinson, Franklin Lakes, NJ, USA) for relative DNA content based on red fluorescence. Cell morphology was analyzed using a light microscope (Zeiss Axiovert 200M; Carl Zeiss, Göttingen, Germany).

Western blot analysis. The cells were washed with cold PBS and lysed on ice in modified RIPA buffer $(50 \mathrm{mM}$ Tris- $\mathrm{HCl}$, $\mathrm{pH} 7,4,1 \%$ NP-40, 0,25\% Na-deoxycholate, $150 \mathrm{mM} \mathrm{NaCl}$, $1 \mathrm{mM} \mathrm{Na} \mathrm{VO}_{4}$, and $1 \mathrm{mM} \mathrm{NaF}$ ) containing protease inhibitors (100 $\mu \mathrm{M}$ phenylmethylsulfonyl fluoride, $10 \mu \mathrm{g} / \mathrm{ml}$ leupeptin, $10 \mu \mathrm{g} / \mathrm{ml}$ pepstatin and $2 \mathrm{mM}$ EDTA). The lysates were centrifuged at $13,000 \times \mathrm{g}$ for $15 \mathrm{~min}$ at $4^{\circ} \mathrm{C}$ and the supernatant fractions were collected. Proteins were separated by SDS-PAGE and transferred onto an immobilon-P membrane (Amersham, Uppsala, Sweden). Specific proteins were detected using enhanced chemiluminescence.

Prostaglandin $E_{2}\left(P_{G} E_{2}\right)$ assay. $\mathrm{PGE}_{2}$ levels in the culture medium were assayed using an enzyme immunoassay kit following the manufacturer's instructions (Cayman Chemical Co.). The assay is based on the competition between peroxidase (or alkaline phosphate)-conjugated tracer $\mathrm{PGE}_{2}$ and $\mathrm{PGE}_{2}$ in the medium for a limited number of $\mathrm{PGE}_{2}$-specific Abs. The amount of remaining tracer $\mathrm{PGE}_{2}$ was determined by the addition of substrates for peroxidase (or alkaline phosphatase). OD values were determined at 405 (or 450) $\mathrm{nm}$, as previously described (21).

Measurement of ROS production. The intracellular accumulation of ROS was determined using the fluorescent probe, 2',7'-dichlorodihydrofluorescein diacetate ( $\left.\mathrm{H}_{2} \mathrm{DCFDA}\right)$. $\mathrm{H}_{2}$ DCFDA is commonly used to measure ROS generation (22). The AMC-HN4 cells were pre-treated with $200 \mu \mathrm{M}$ trolox and $500 \mu \mathrm{M}$ MnTBAP for $30 \mathrm{~min}$, and the cells were then incubated with $3 \mu \mathrm{M}$ withaferin A for $30 \mathrm{~min}$. The cells were stained with the fluorescent dye, $\mathrm{H}_{2} \mathrm{DCFDA}$, for an additional $10 \mathrm{~min}$. Subsequently, the cells were trypsinized and resuspended in PBS, and fluorescence was measured at specific time intervals with a flow cytometer (Becton-Dickinson) or was detected using a fluorescence microscope (Zeiss, Goettingen, Germany).
Statistical analysis. The data were analyzed using one-way ANOVA and post-hoc comparisons (Student-Newman-Keuls test) using the Statistical Package for Social Sciences 22.0 software (SPSS Inc., Chicago, IL, USA).

\section{Results}

Withaferin $A$ induces apoptosis and COX-2 expression in the human head and neck carcinoma cells, AMC-HN4. We have previously reported that withaferin A induces apoptosis in human renal carcinoma Caki cells (23) and human leukemia U937 cells (8). In this study, to determine whether withaferin A induces apoptosis, AMC-HN4 cells were treated with the indicated concentrations of withaferin A for $24 \mathrm{~h}$. FACS analysis for the measurement of DNA content and western blot analysis for the detection of the cleavage of PARP, a substrate of caspase-3, were performed. Withaferin A markedly increased the sub-G1 population and the cleavage of PARP in a doseand time-dependent manner (Fig. 1). COX-2-overexpressing cancer cells are resistant to anticancer drug-mediated apoptosis (24). Therefore, we examined whether withaferin A increases COX-2 expression in the AMC-HN4 cells. As shown in Fig. 1, withaferin A increased COX-2 expression in a doseand time-dependent manner.

Subsequently, we investigated whether the withaferin Ainduced expression of COX-2 is a consequence of apoptosis. Treatment with the pan-caspase inhibitor, z-VAD-fmk (z-VAD), markedly inhibited the withaferin A-induced increase in the sub-G1 population and the cleavage of PARP (Fig. 2). However, the withaferin A-induced increase in the expression COX-2 was not affected by treatment with z-VAD. Therefore, these data indicate that the withaferin A-mediated COX-2 expression is not a consequence of apoptosis.

Withaferin A-induced COX-2 expression has no effect on apoptosis. Previous studies have reported that the downregulation of COX-2 expression and the inhibition of $\mathrm{PGE}_{2}$ production enhances anticancer drug-mediated apoptosis $(19,20,22,23,25)$. Therefore, we examined whether the inhibition of $\mathrm{PGE}_{2}$ production increases withaferin A-mediated apoptosis. NS-398, a COX-2 inhibitor, markedly blocked the withaferin A-mediated production of $\mathrm{PGE}_{2}$ (Fig. 3A). However, the withaferin A-induced increase in the sub-G1 population and the cleavage of PARP were not affected by NS-398 treatment (Fig. 3B). These data indicate that the withaferin A-induced expression of COX-2 is not associated with apoptosis in the head and neck carcinoma cells, AMC-HN4.

Withaferin A-mediated apoptosis is independent of ROS signaling. Withaferin A has been shown to increase ROS production, and ROS is involved in apoptosis (5). Therefore, we investigated whether withaferin A increases intracellular ROS levels in AMC-HN4 cells. Withaferin A markedly increased intracellular ROS production (Fig. 4A). Subsequently, we wished to dertermine whether ROS are involved in withaferin A-induced apoptosis. The ROS scavengers, trolox and MnTBAP, inhibited withaferin A-mediated ROS production (Fig.4A); however, the sub-G1 population and the cleavage of PARP were not affected (Fig. 4B). Furthermore, the induction of COX-2 expression by withaferin A was independent of 

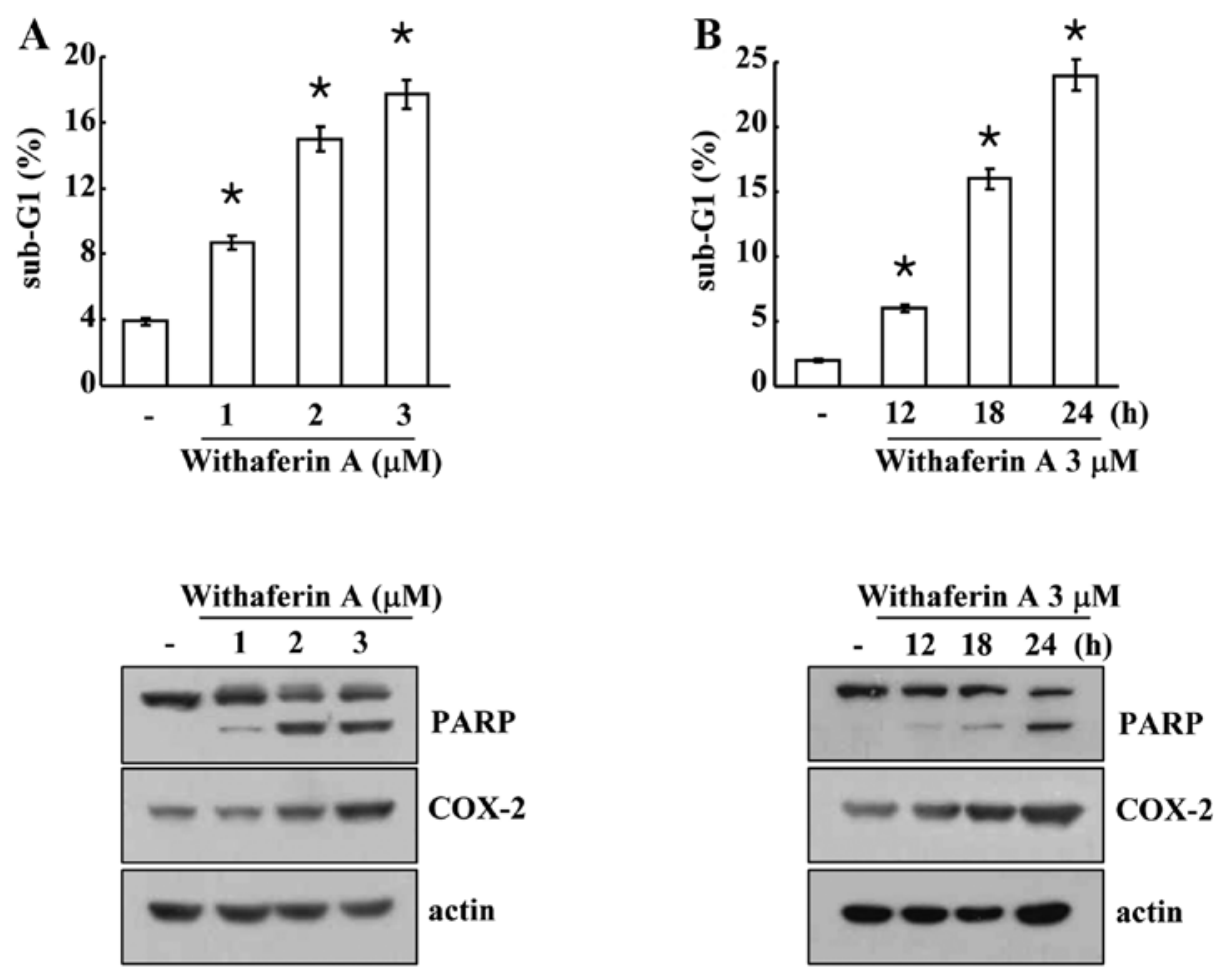

Figure 1. Withaferin A induces apoptosis and upregulates cyclooxygenase-2 (COX-2) expression in AMC-HN4 cells. (A) AMC-HN4 cells were treated with the indicated concentrations of withaferin A for $24 \mathrm{~h}$. (B) AMC-HN4 cells were treated with withaferin A ( $3 \mu \mathrm{M})$ for the indicated perios of time. The sub-G1 population was analyzed by fluorescence activated cell sorting (FACS) (upper panels). Equal amounts of cell lysates ( $60 \mu \mathrm{g})$ were separated by gel electrophoresis and analyzed by western blotting for PARP and COX-2 protein. Actin served as a control for protein loading (lower panels). The values in (A and B) represent the means \pm SD from 3 independent samples. " $p<0.05$ compared to the control (untreated cells).
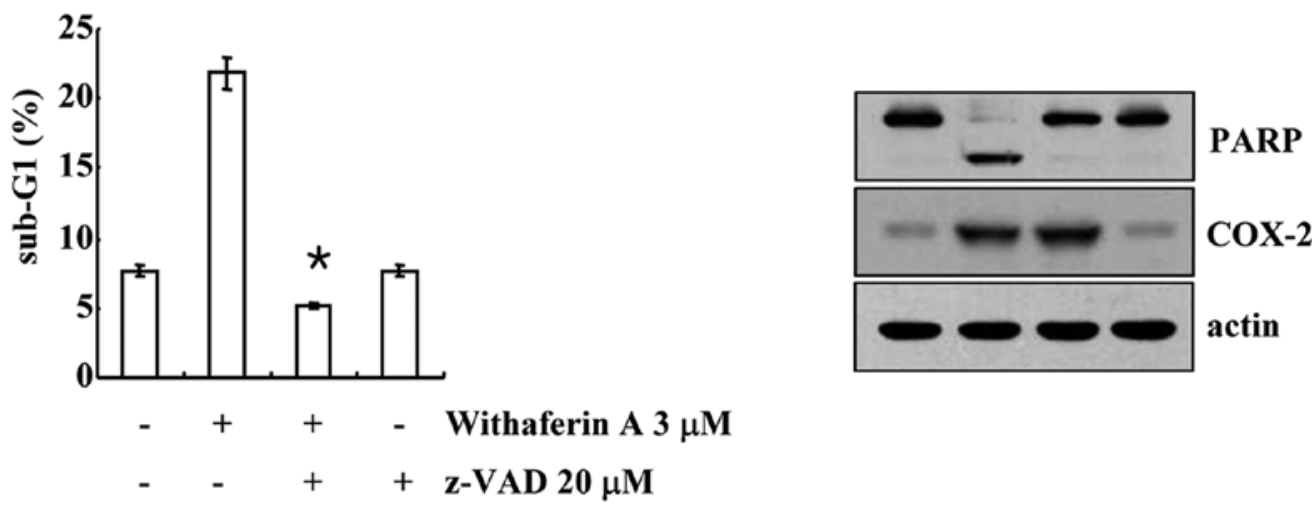

Figure 2. Upregulation of cyclooxygenase-2 (COX-2) expression is not a consequence of apoptosis in withaferin A-treated AMC-HN4 cells. AMC-HN4 cells were treated with $20 \mu \mathrm{M} \mathrm{z}$-VAD-fmk (z-VAD; a pan-caspase inhibitor) for 30 min prior to treatment with withaferin A ( $3 \mu \mathrm{M})$ for $24 \mathrm{~h}$. The sub-G1 population was analyzed by fluorescence activated cell sorting (FACS) (left panel). Equal amounts of cell lysates (60 $\mu \mathrm{g}$ ) were separated by gel electrophoresis and analyzed by western blotting for PARP and COX-2 protein. Actin served as a control for protein loading (right panel). ${ }^{\mathrm{p}}<0.05$ compared to treatment with withaferin A alone.

ROS production (Fig. 4B). These data indicate that ROS is not associated with withaferin A-mediated apoptosis.

Withaferin A-mediated apoptosis is reversed by thiol donors. Previous studies have reported that excess amounts of thiol donors block the effects of withaferin A $(26,27)$. To determine whether thiol donors inhibit withaferin A-induced apoptosis, AMC-HN4 cells were treated with NAC and DTT. Both thiol donors markedly inhibited morphological changes in the withaferin A-treated cells (Fig. 5A). Furthermore, NAC and
DTT blocked the increase in the sub-G1 population and the cleavage of PARP (Fig. 5B). These data suggest that the mechanism of thiol oxidation is important for withaferin A-mediated apoptosis.

\section{Discussion}

In the present study, we demonstrated that withaferin A induced apoptosis in the human head and neck carcinoma cells, AMC-HN4. Withaferin A increased COX-2 expres- 

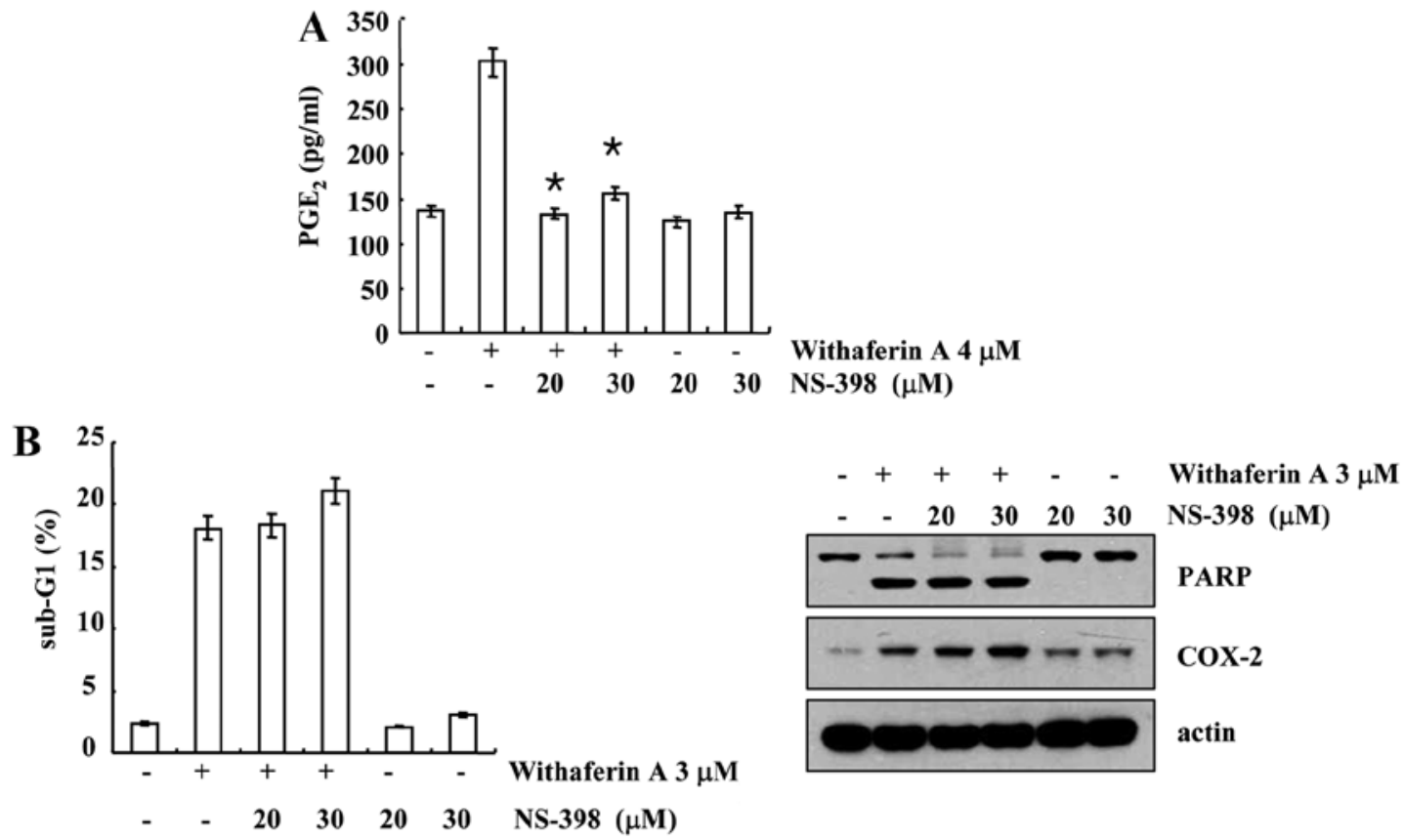

Figure 3. Upregulation of cyclooxygenase-2 (COX-2) expression is not associated with withaferin A-induced apoptosis in AMC-HN4 cells. (A and B) AMC-HN4 cells were treated with the indicated concentrations of NS-398 for $30 \mathrm{~min}$ prior to treatment with withaferin A ( $3 \mu \mathrm{M})$ for $24 \mathrm{~h}$. (A) $\mathrm{PGE}_{2}$ production was determined using an enzyme immunoassay kit. (B) The sub-G1 population was analyzed by fluorescence activated cell sorting (FACS) (left panel). Equal amounts of cell lysates $(60 \mu \mathrm{g})$ were separated by gel electrophoresis and analyzed by western blotting for PARP and COX-2 protein. (B) Actin served as a control for protein loading (right panel). $\mathrm{p}<0.05$ compared to treatment with withaferin A alone.

A
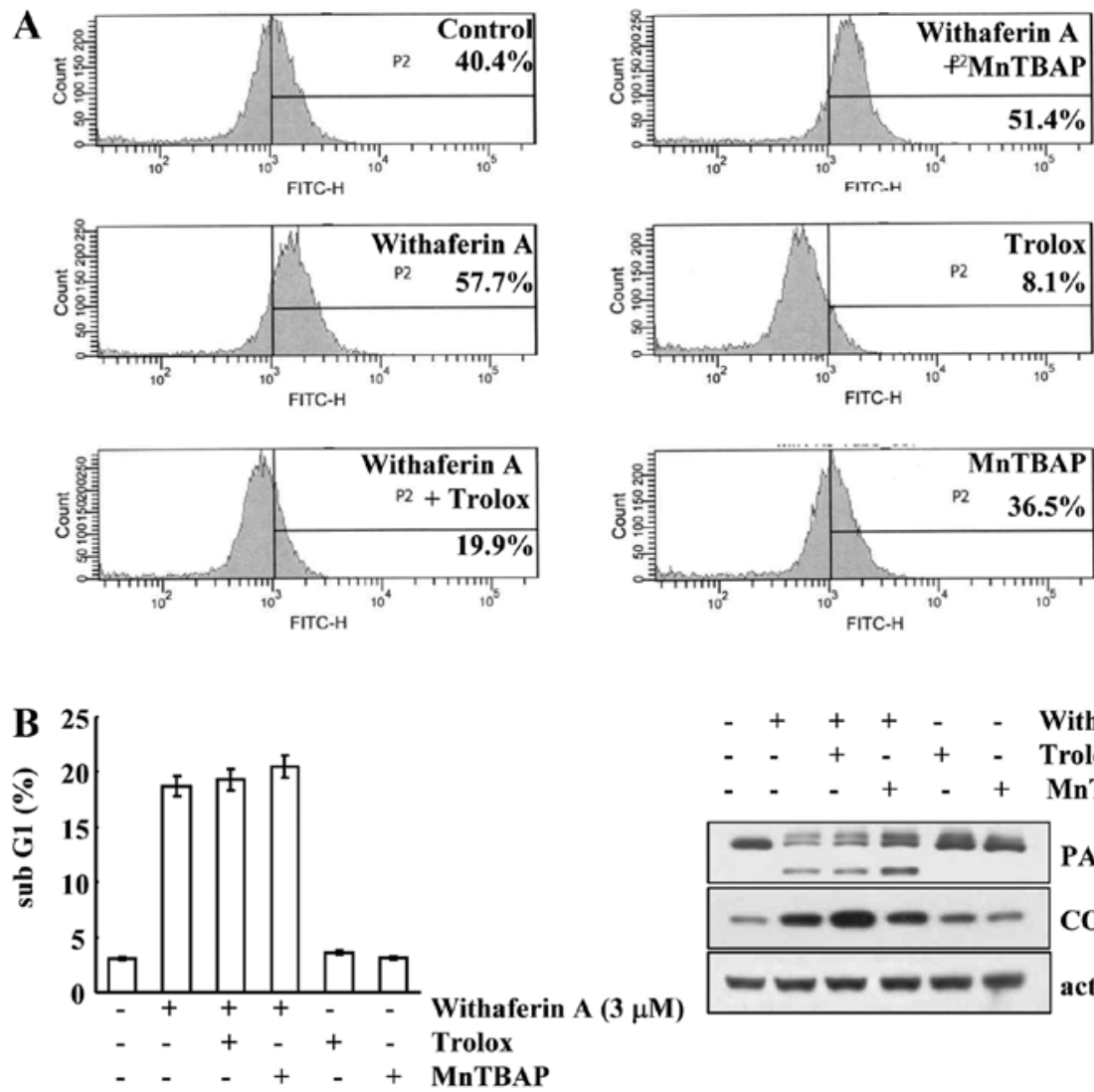

$-\quad+\quad+\quad-\quad-$ Withaferin A $(3 \mu \mathrm{M})$

$-\quad+-+-$ Trolox

$-\quad-+-+\operatorname{MnTBAP}$

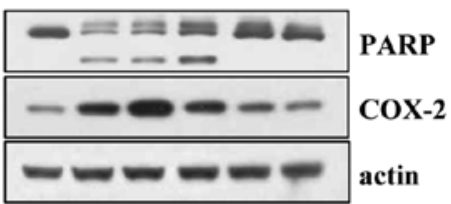

Figure 4. Reactive oxygen species (ROS) production is not involved in withaferin A-mediated apoptosis and the upregulation of cyclooxynase-2 (COX-2) expression in AMC-HN4 cells. (A) AMC-HN4 cells were treated with trolox (200 M) and MnTBAP (500 $\mu \mathrm{M})$ for 30 min prior to treatment with withaferin A $(3 \mu \mathrm{M})$ for $30 \mathrm{~min}$ and then stained with the fluorescent dye, $\mathrm{H}_{2} \mathrm{DCFDA}$, for an additional 10 min. Fluorescence was immediately assayed by flow cytometry. (B) AMC-HN4 cells were treated with trolox $(200 \mu \mathrm{M})$ and MnTBAP $(500 \mu \mathrm{M})$ for 30 min prior to treatment with withaferin A $(3 \mu \mathrm{M})$ for 24 h. The sub-G1 population was analyzed by fluorescence activated cell sorting (FACS) (left panel). Equal amounts of cell lysates (60 $\mu \mathrm{g}$ ) were separated by gel electrophoresis and analyzed by western blot analysis for PARP and COX-2 protein. Actin served as a control for protein loading (right panel). 
A
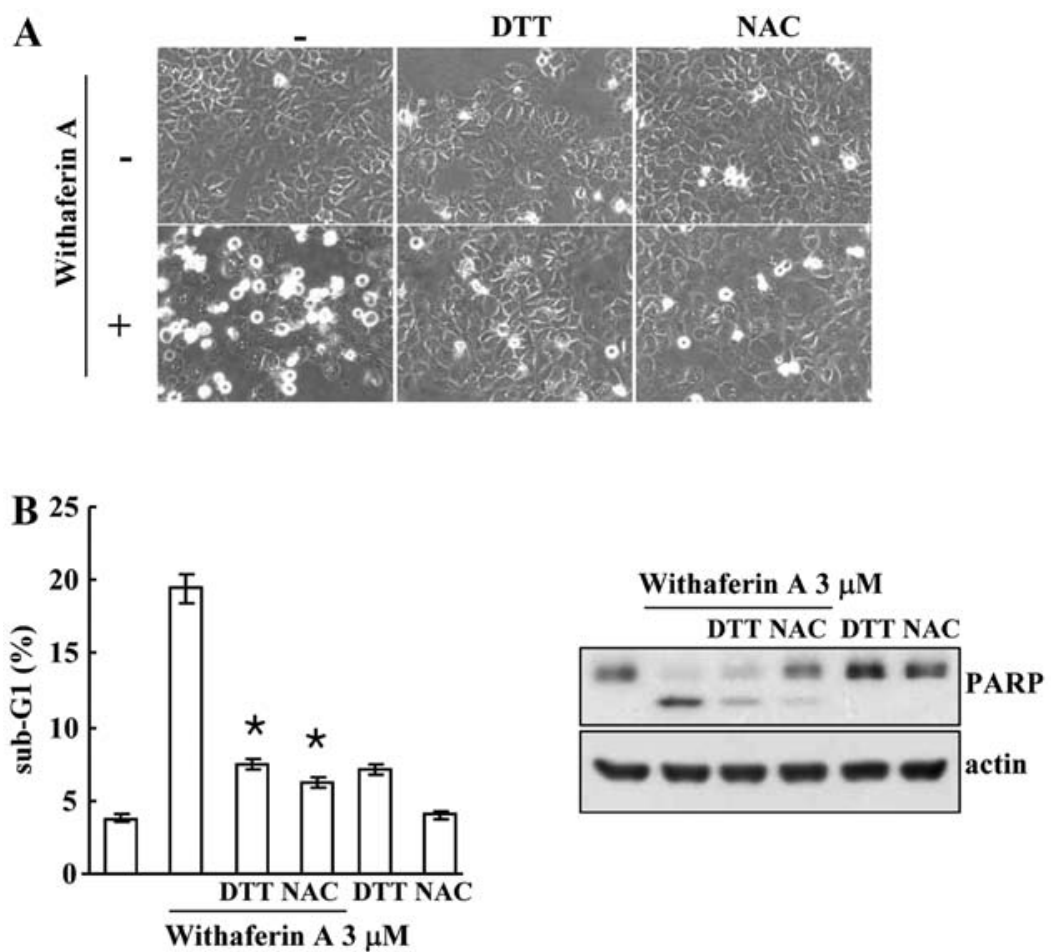

Figure 5. Effects of thiol oxidation on withaferin A-induced apoptosis of AMC-HN4 cells. (A and B) AMC-HN4 cells were treated with dithiothreitol (DTT, $2 \mathrm{mM}$ ) and $\mathrm{N}$-acetylcysteine (NAC, $5 \mathrm{mM}$ ) for 30 min prior to treatment with withaferin A ( $3 \mu \mathrm{M})$ for $24 \mathrm{~h}$. (A) Cell morphology was examined using interference light microscopy. (B) The sub-G1 population was analyzed by fluorescence activated cell sorting (FACS) (left panel). Equal amounts of cell lysates (60 $\mu \mathrm{g}$ ) were separated by gel electrophoresis and analyzed by western blotting for PARP and cyclooxygenase-2 (COX-2) protein. (B) Actin served as a control for protein loading (right panel). " $\mathrm{p}<0.05$ compared to treatment with withaferin A alone. The data represent 3 independent experiments.

sion and $\mathrm{PGE}_{2}$ production, but $\mathrm{PGE}_{2}$ was not associated with apoptosis. Furthermore, the upregulation of intracellular ROS had no effect on apoptosis. Thiol donors only markedly inhibited withaferin A-mediated apoptosis. Therefore, our results suggest that thiol oxidation plays an important role in withaferin A-mediated apoptosis.

COX-2 was overexpressed and the levels of PG, such as $\mathrm{PGE}_{2}$, were increased in the cancer cells. Previous studies have reported that $\mathrm{COX}-2$ induces proliferation, angiogenesis, migration and invasion, and inhibits apoptosis. A selective COX-2 inhibitor (celecoxib) has been shown to induce apoptosis in prostate carcinoma (25), colon carcinoma (28), cholangiocarcinoma (29), pancreatic carcinoma and melanoma (30) cells. Furthermore, the overexpression of COX-2 reduces apoptosis. Sun et al (31) reported that COX-2 overexpression inhibits the release of cytochrome $c$ and caspase activation in colon carcinoma cells treated with COX-2 inhibitor and 5-fluorouracil. By contrast, the overexpression of COX-2 in osteosarcoma cells has been shown to decrease cell viability (32). Xu et al (32) reported that apoptosis by COX-2-overexpression is independent of $\mathrm{PGE}_{2}$, whereas the inhibition of ROS production reduces apoptosis in osteosarcoma cells. In head and neck carcinoma, celecoxib and sulindac have been shown to reduce proliferation and induce apoptosis (33). Furthermore, the inhibition of COX-2 enhances sensitivity to anticancer drugs, such as doxorubicin, vincristine, cisplatin, bleomycin and 5-fluorouracil (33). Therefore, we hypothesized that the withaferin A-induced expression of COX-2 and $\mathrm{PGE}_{2}$ production enhances resistance to apoptosis. However, although NS-398 (a COX-2 inhibitor) markedly blocked withaferin A-mediated $\mathrm{PGE}_{2}$ production, apoptosis was not affected (Fig. 3B). Therefore, the role of COX-2 in anticancer effects is dependent on cell type and cell conditions.

Withaferin A exerts pro-apoptotic (4-8), anti-proliferative (34), anti-angiogenic (35), and anti-invasive (36) effects through multiple mechanisms. Among these, the upregulation of intracellular ROS is important for withaferin A-mediated apoptosis (37). We also detected withaferin A-mediated ROS production in AMC-HN4 cells (Fig. 4A). ROS scavengers (trolox and MnTBAP) reduced ROS production in the withaferin A-treated cells, whereas the sub-G1 population and the cleavage of PARP were not affected (Fig. 4B). Thiol oxidation is important for the function of withaferin A. Withaferin A inhibits I $\mathrm{B}$ kinase- $\beta$ activity, and DTT reverses the inhibitory effects (27). Furthermore, withaferin A-mediated apoptosis is reversed by DTT in erythromyelogenous leukemia cells (26). In this study, the thiol donors, DTT and NAC, markedly blocked withaferin A-mediated apoptosis (Fig. 5B). Withaferin A has $\alpha, \beta$-unsaturated ketone moiety in the $\mathrm{A}$ ring, which reacts with protein thiol nucleophiles (38). Therefore, withaferin A may target cysteine residues of proteins, such as kinases, phosphatases and chaperons. Therefore, further studies are required to identify the target proteins of withaferin A in head and neck carcinoma cells.

Taken together, our results suggest that the withaferin A-mediated apoptosis is independent of COX-2 expression and ROS production. Thiol oxidation is an important mechanisms of withaferin A-induced apoptosis in head and neck carcinoma cells. 


\section{References}

1. Srinivasan S, Ranga RS, Burikhanov R, Han SS and Chendil D Par-4-dependent apoptosis by the dietary compound withaferin A in prostate cancer cells. Cancer Res 67: 246-253, 2007.

2. Malik F, Kumar A, Bhushan S, et al: Reactive oxygen species generation and mitochondrial dysfunction in the apoptotic cell death of human myeloid leukemia HL-60 cells by a dietary compound withaferin A with concomitant protection by $\mathrm{N}$-acetyl cysteine. Apoptosis 12: 2115-2133, 2007.

3. Stan SD, Hahm ER, Warin R and Singh SV: Withaferin A causes FOXO3a- and Bim-dependent apoptosis and inhibits growth of human breast cancer cells in vivo. Cancer Res 68: 7661-7669, 2008

4. Choi MJ, Park EJ, Min KJ, Park JW and Kwon TK: Endoplasmic reticulum stress mediates withaferin A-induced apoptosis in human renal carcinoma cells. Toxicol In Vitro 25: 692-698, 2011

5. Mayola E, Gallerne C, Esposti DD, et al: Withaferin A induces apoptosis in human melanoma cells through generation of reactive oxygen species and down-regulation of Bcl-2. Apoptosis 16 : 1014-1027, 2011.

6. Hahm ER, Moura MB, Kelley EE, Van Houten B, Shiva S and Singh SV: Withaferin A-induced apoptosis in human breast cancer cells is mediated by reactive oxygen species. PLoS One 6 : e23354, 2011.

7. Mandal C, Dutta A, Mallick A, Chandra S, Misra L and Sangwan RS: Withaferin A induces apoptosis by activating p38 Mitogen-activated protein kinase signaling cascade in leukemic cells of lymphoid and myeloid origin through mitochondrial death cascade. Apoptosis 13: 1450-1464, 2008.

8. Oh JH, Lee TJ, Kim SH, et al: Induction of apoptosis by withaferin A in human leukemia U937 cells through down-regulation of Akt phosphorylation. Apoptosis 13: 1494-1504, 2008.

9. Samadi AK, Tong X, Mukerji R, Zhang H, Timmermann BN and Cohen MS: Withaferin A, a cytotoxic steroid from Vassobia breviflora, induces apoptosis in human head and neck squamous cell carcinoma. J Nat Prod 73: 1476-1481, 2010.

10. Tanabe T and Tohnai N: Cyclooxygenase isozymes and their gene structures and expression. Prostaglandins Other Lipid Mediat 68-69: 95-114, 2002.

11. Tsuji S, Tsujii M, Kawano S and Hori M: Cyclooxygenase-2 upregulation as a perigenetic change in carcinogenesis. J Exp Clin Cancer Res 20: 117-129, 2001.

12. Ledwith BJ, Pauley CJ, Wagner LK, Rokos CL, Alberts DW and Manam S: Induction of cyclooxygenase-2 expression by peroxisome proliferators and non-tetradecanoylphorbol 12,13-myristate-type tumor promoters in immortalized mouse liver cells. J Biol Chem 272: 3707-3714, 1997.

13. Okami J, Yamamoto H, Fujiwara Y, et al: Overexpression of cyclooxygenase-2 in carcinoma of the pancreas. Clin Cancer Res 5: 2018-2024, 1999.

14. Eberhart CE, Coffey RJ, Radhika A, Giardiello FM, Ferrenbach S and DuBois RN: Up-regulation of cyclooxygenase 2 gene expression in human colorectal adenomas and adenocarcinomas. Gastroenterology 107: 1183-1188, 1994.

15. Ryu HS, Chang KH, Yang HW, Kim MS, Kwon HC and Oh KS: High cyclooxygenase-2 expression in stage IB cervical cancer with lymph node metastasis or parametrial invasion. Gynecol Oncol 76: 320-325, 2000 .

16. Hara S, Kondo Y, Matsuzawa I, et al: Expression of cycloxygenase-2 in human bladder and renal cell carcinoma. Adv Exp Med Biol 507: 123-126, 2002.

17. Celenk F, Bayramoglu I, Yilmaz A, Menevse A and Bayazit Y: Expression of cyclooxygenase-2, 12-lipoxygenase, and inducible nitric oxide synthase in head and neck squamous cell carcinoma. J Craniofac Surg 24: 1114-1117, 2013.

18. Hara A, Yoshimi N, Niwa M, Ino N and Mori H: Apoptosis induced by NS-398, a selective cyclooxygenase-2 inhibitor, in human colorectal cancer cell lines. Jpn J Cancer Res 88 : 600-604, 1997.

19. Sawaoka H, Kawano S, Tsuji S, et al: Cyclooxygenase-2 inhibitors suppress the growth of gastric cancer xenografts via induction of apoptosis in nude mice. Am J Physiol 274: G1061-1067, 1998.
20. Limami Y, Pinon A, Leger DY, et al: HT-29 colorectal cancer cells undergoing apoptosis overexpress COX-2 to delay ursolic acid-induced cell death. Biochimie 93: 749-757, 2011.

21. Yang MS, Ji KA, Jeon SB, et al: Interleukin-13 enhances cyclooxygenase-2 expression in activated rat brain microglia: implications for death of activated microglia. J Immunol 177: 1323-1329, 2006

22. LeBel CP, Ischiropoulos $\mathrm{H}$ and Bondy SC: Evaluation of the probe 2',7'-dichlorofluorescin as an indicator of reactive oxygen species formation and oxidative stress. Chem Res Toxicol 5: 227-231, 1992.

23. Um HJ, Min KJ, Kim DE and Kwon TK: Withaferin A inhibits JAK/STAT3 signaling and induces apoptosis of human renal carcinoma Caki cells. Biochem Biophys Res Commun 427: 24-29, 2012.

24. Liu B, Qu L and Tao H: Cyclo-oxygenase 2 up-regulates the effect of multidrug resistance. Cell Biol Int 34: 21-25, 2009.

25. Hsu AL, Ching TT, Wang DS, Song X, Rangnekar VM and Chen CS: The cyclooxygenase-2 inhibitor celecoxib induces apoptosis by blocking Akt activation in human prostate cancer cells independently of Bcl-2. J Biol Chem 275: 11397-11403, 2000.

26. Suttana W, Mankhetkorn S, Poompimon W, et al: Differential chemosensitization of P-glycoprotein overexpressing K562/Adr cells by withaferin A and Siamois polyphenols. Mol Cancer 9: 99, 2010.

27. Kaileh M, Vanden Berghe W, Heyerick A, et al: Withaferin A strongly elicits IkappaB kinase beta hyperphosphorylation concomitant with potent inhibition of its kinase activity. J Biol Chem 282: 4253-4264, 2007

28. Arico S, Pattingre S, Bauvy C, et al: Celecoxib induces apoptosis by inhibiting 3-phosphoinositide-dependent protein kinase-1 activity in the human colon cancer HT-29 cell line. J Biol Chem 277: 27613-27621, 2002

29. Wu GS, Zou SQ, Liu ZR, Tang ZH and Wang JH: Celecoxib inhibits proliferation and induces apoptosis via prostaglandin E2 pathway in human cholangiocarcinoma cell lines. World J Gastroenterol 9: 1302-1306, 2003.

30. Wu G, Yi J, Di F, Zou S and Li X: Celecoxib inhibits proliferation and induces apoptosis via cyclooxygenase-2 pathway in human pancreatic carcinoma cells. J Huazhong Univ Sci Technolog Med Sci 25: 42-44, 2005.

31. Sun Y, Tang XM, Half E, Kuo MT and Sinicrope FA: Cyclooxygenase-2 overexpression reduces apoptotic susceptibility by inhibiting the cytochrome $c$-dependent apoptotic pathway in human colon cancer cells. Cancer Res 62: 6323-6328, 2002.

32. Xu Z, Choudhary S, Voznesensky O, et al: Overexpression of COX-2 in human osteosarcoma cells decreases proliferation and increases apoptosis. Cancer Res 66: 6657-6664, 2006.

33. Hashitani S, Urade M, Nishimura N, et al: Apoptosis induction and enhancement of cytotoxicity of anticancer drugs by celecoxib, a selective cyclooxygenase-2 inhibitor, in human head and neck carcinoma cell lines. Int J Oncol 23: 665-672, 2003.

34. Cai Y, Sheng ZY, Chen Y and Bai C: Effect of Withaferin A on A549 cellular proliferation and apoptosis in non-small cell lung cancer. Asian Pac J Cancer Prev 15: 1711-1714, 2014.

35. Mohan R, Hammers HJ, Bargagna-Mohan P, et al: Withaferin A is a potent inhibitor of angiogenesis. Angiogenesis 7: 115-122, 2004.

36. Thaiparambil JT, Bender L, Ganesh T, et al: Withaferin A inhibits breast cancer invasion and metastasis at sub-cytotoxic doses by inducing vimentin disassembly and serine 56 phosphorylation. Int J Cancer 129: 2744-2755, 2011.

37. Sen N, Banerjee B, Das BB, et al: Apoptosis is induced in leishmanial cells by a novel protein kinase inhibitor withaferin A and is facilitated by apoptotic topoisomerase I-DNA complex. Cell Death Differ 14: 358-367, 2007.

38. Fuska J, Fuskova A, Rosazza JP and Nicholas AW: Novel cytotoxic and antitumor agents. IV. Withaferin A: relation of its structure to the in vitro cytotoxic effects on P388 cells. Neoplasma 31: 31-36, 1984. 